\title{
Interpreters of Nineteenth Century Viewed by Anthropologists
}

\author{
Christina Lachat Leal \\ Departamento de Traducción e Interpretación \\ Universidad de Granada (Spain) \\ clachat@ugr.es
}

\section{Doi:10.5901/mjss.2013.v4n9p284}

\begin{abstract}
Along the history the interpreters were discreet witnesses of important historical events. We discovered that the visual analysis of photographs is an extremely useful instrument for research in interpretation which enables us to discover how the interpreter was perceived. In our research on the history of interpretation in pictures, we created a database with nearly 350 photographs of interpreters since 1846 until 1939 available in digital collections. We must acknowledge that the higher number of photographs has us a little surprised, and after a first analysis we discovered that photographers who realised most portraits worked for anthropological institutions or were themselves anthropologists. Our aim is to carry out a visual analysis of these portraits to discover, if the concept of the Other of the anthropologists of this period changes in liaison with the interpreters. Also we analyze eleven portraits of four interpreters by re-contextualising them. The results suggest that the interpreters are seen like specimens. The anthropologists do not see the interpreters; they perceive only the Other savage.
\end{abstract}

Keywords: interpreter, photography, visual anthropology, history of interpretation, otherness

\section{Introduction}

Au long de l'histoire, les interprètes ont été des témoins discrets d'importants événements historiques. Si discrets que, sauf exception, peu de personnes, mis à part les chercheurs, peuvent en nommer. Quoiqu'il existe quelques documents historiques où figure le nom de l'interprète ou sa participation, ceux-ci y dédient peu de lignes. Mais récemment, nous avons découvert que les documents visuels et leur analyse nous permettraient, d'une part, de reconstruire la situation de l'interprétation, et d'autre part, de découvrir comment était perçu l'interprète.

Pour notre recherche sur l'histoire de l'interprétation en images photographiques, nous avons commencé par la création d'une base de données de photographies disponibles dans les collections digitales datant de 1846 jusqu'à 1939 (Lachat Leal, 2013). Aujourd'hui, nous avons recensé près de 350 photographies dont 229 portraits. Nous devons avouer que le nombre élevé de portraits nous a un peu surpris, et après une première analyse, nous avons découvert que 151 de ces portraits avaient été pris par des photographes qui travaillaient pour des institutions anthropologiques ou étaient eux-mêmes anthropologues. II faut signaler que dès le premier moment ces portraits nous ont, d'une certaine façon, troublés ce qui nous a poussés à entreprendre cette étude.

D'une part, selon Bourdieu (1965:24) chaque photographie exprime « outre les intentions de celui qui l'a faite, le système de perception, de pensée et d'appréciation commun à tout un groupe ». D'autre part, "la production photographique des anthropologues au XIXe siècle fut d'ailleurs particulièrement conséquente et concerne essentiellement une approche " physique " de l'Autre » (Jehel, 2000:47). Donc notre principal objectif sera de déchiffrer «le surplus de signification que trahit» (Bourdieu et al., 1965:25) ces portraits, et comment la communauté des anthropologues du XIXe siècle percevait l'interprète.

\section{Photographie et anthropologie au XIXe siècle}

Au XIXe siècle l'anthropologie qui s'était proposé de classer systématiquement les différents « types humains », aspirait à devenir une science. Aussi devait-elle se munir de méthodes et d'instruments dotés de qualités scientifiques reconnues. Étant donner que les anthropologues étaient alors essentiellement des compilateurs, ils avaient besoin de représentations « en images des individus qu'il était impossible de rapporter comme échantillon » (Maresca, 1996: 124125). La photographie ayant à l'époque la considération d'empreinte exacte et fidèle de la réalité, contrairement aux dessins et croquis considérés subjectifs, fut rapidement adoptée par les scientifiques comme Charles Darwin et les 
anthropologues. En 1854, l'Association britannique pour le progrès de la science publie un manuel avec des instructions pour les voyageurs, consuls et résidents où on leur conseille de prendre des portraits photographiques individuels ( $A$ Manual of Ethnological Inquiry, 1854:195). Une fois choisi l'instrument scientifique, il ne restait qu'à élaborer une méthode.

\subsection{Le portrait anthropométrique}

Très vite, les anthropologues découvrent que pas toutes les photographies servent à leurs études. Le médecin et anthropologue Paul Broca publie en 1865 les Instructions générales pour les recherches et observations anthropologiques où il développe une méthode anthropométrique, et qui recueillent des instructions pour la photographie anthropologique: «On reproduira par la photographie : $1^{0}$ des têtes nues qui devront toujours, sans exception, être prises exactement de face ou exactement de profil [...]. Les portraits en pied avec l'accoutrement caractéristique de la tribu ont aussi leur importance »(Broca, 1865: 6).

Un peu plus tard, le naturaliste et photographe Eugène Trutat donnera des instructions assez précises qui permettraient de comparer à «première vue des spécimens recueillis dans de différents pays » (1892: 5) sur la dimension, l'illumination et la prise de vue des portraits :

Pour les portraits de face, il est important de placer très exactement le sujet, de telle sorte que les deux côtés de la face soient vus également; il suffit pour arriver au résultat voulu, de voir également les deux oreilles : j'avoue qu'alors on arrive souvent à des effets disgracieux, mais il importe avant tout d'obtenir des documents scientifiques [...] Les sujets dont on prendra les portraits de face ou de profil pourront être photographiés avec ou sans vêtements : dans le primer cas il faudra chercher le plus possible à dégagé le cou, afin de donner aux contours de la tête toute sa valeur (Trutat, 1892: 6-7).

Comme on le voit le portrait devient un instrument anthropométrique froid et impersonnel pour comparer et classer les individus en catégorie raciale. La plupart de ces portraits sont très laids, désagréables et même grossiers, et c'est la raison pour laquelle ils ont attiré notre attention.

\subsection{Les photographes}

Il est impossible de réaliser une analyse visuelle de photographies sans les contextualiser. Pour ce faire, nous allons présenter une brève note biographique des auteurs, les photographes.

\subsubsection{De Lancey W. Gill (1859-1940)}

Photographe et peintre du Bureau of American Ethnology pendant près de soixante ans, on lui demanda de photographier les délégations de chefs indiens qui visitaient Washington (Scherer, 2006: 54). Ses portraits des membres de délégations auraient pu être honorables, mais malheureusement ils les présentent comme des spécimens scientifiques (Scherer, 2006: 57).

\subsubsection{William Dinwiddie (1867-1934)}

Pendant dix ans, il travailla, lui aussi, pour le Bureau of American Ethnology. En 1896, il décida de changer de carrière, et il deviendra un reporter de guerre reconnu (Leonard y Marquis, 1898:198). II est l'auteur de treize portraits de notre étude qui sont un exemple de froideur scientifique.

\subsubsection{Wells Moses Sawyer (1863-1960)}

Wells Sawyer, peintre reconnu, travailla comme photographe pour le Bureau of American Ethnology en 1897. II fut chargé de photographier les membres des délégations indiennes. Ses portraits sont impersonnels, antipathiques avec la tête floue, la posture du corps raide et les yeux non-focalisés semblent regarder au-delà du photographe (Scherer, 2006: 55). 


\subsubsection{Jacques-Philippe Potteau (1807-1876)}

Ce photographe français travailla pour le Musée d'Histoire Naturelle de Paris pour lequel il réalisa près de 550 portraits (Sheptytsky-Zäll, 2009). « II entreprend la série des ambassades qui consiste à photographier systématiquement tout le personnel des ambassades en visite à Paris de passage au Muséum d'histoire naturelle » (Jehel, 1995:36). Quoiqu'il se qualifie d'artiste ses photographies ont un but nettement scientifique.

\subsubsection{Frank Albert Rinehart (1861-1928)}

Le cas de Frank Albert Rinehart est différent, car il ne travaillait pas pour une institution anthropologique, ni était anthropologue. II avait un studio de photographie à Omaha, Nebraska et en 1898, il fut engagé comme photographe officiel de l'Exposition Internationale de Trans-Mississippi (Smithsonian American Art Museum). Donc, son intention n'était pas de produire des documents scientifiques mais des documents historiques sur les Indiens d'Amérique. Ses portraits sont une antithèse dans notre étude.

\subsubsection{Maurice Vidal Portman (1860-1935)}

Nous ne pouvions pas finir ce petit parcours biographique sur les photographes sans mentionner Portman, anthropologue autodidacte, auteur d'une ample étude photographique de onze tomes sur les habitants des îles Andaman (Brisset 1999). Dans notre base de données nous n'avons qu'une seule photographie de Portman, celle de l'interprète Riwa ${ }^{1}$, cependant nous avons considéré qu'on devait l'inclure dans ce travail, parce que, d'une part, c'est un anthropologue très cité dans les études d'anthropologies visuelles, et d'autre part, car nous pensons qu'il poussa jusqu'à l'extrême la méthode anthropométrique. Lui-même a écrit que les sujets devaient poser avec le corps droit, complètement nu sur un fond d'échiquier noir et blanc, photographiant toutes les malformations (Portman, 1896:76). Mais ce n'est pas tout, il accompagnait ces photos de commentaires sur le tempérament, métier et aspects physiques, entre autres, des sujets. Sur l'interprète Riwa, il écrit :

Riwa, a man of about forty-four, is described [by Portman] as "very intelligent. Government Interpreter for the North Andaman Group of Tribes. Fond of gaiety and dancing. Violent tempered, and hectoring disposition. Penis and left testicle normal. Right testicle small and atrophied. Very lustful. Slight offensive smell from body and breath. Temperament nervous-sanguine" (Sen 2009: 374).

\section{Interprètes et anthropologues}

La relation entre les interprètes et les anthropologues est aussi vieille que l'anthropologie, car ces derniers, soit de forme directe sur le terrain, soit à travers de voyageurs, explorateurs ou photographes, ont le besoin de se communiquer avec les sujets pour connaître leurs mœurs et coutumes. Mais il faut dire que dans les écrits d'anthropologie que l'on connaît, on ne mentionne que rarement à l'interprète, si ce n'est comme informateur.

Dû à la longueur limitée de cette communication et aussi aux droits de reproduction de certaines images, nous n'analyserons que huit portraits de quatre interprètes : un Arapaho, deux Sioux et un Japonais. Mais nous considérons cet échantillon très significatif.

\subsection{Hitouunnen, Cleaver Warden}

Hitouunnen, un Arapaho plus connu comme Cleaver Warden, né vers 1867, fut un des premiers Arapaho qui assista à l'école indienne Carlisle. Il démontra être très doué pour l'écriture et la recherche comme rédacteur du journal de l'école. Quand il retourna à sa réserve, il commença à travailler comme interprète. Plus tard, il travailla pour le Field Museum of Natural History avec les anthropologues Dorsey, Krober et Michelson (Anderson, 1997: XIV). Selon l'anthropologue Anderson, Dorsey et Krober dépendaient tellement de Warden comme collaborateur, interprète et chercheur qu'il devrait être considéré comme co-auteur de leur livre intitulé Traditions of the Arapaho (1997: V).

Nous allons analyser trois portraits de Warden, de trois photographes différents. Nous pouvons classer le premier portrait, celui que William Dinwiddie lui a fait en 1895, comme purement anthropométrique. La position du corps est

1 Pour voir la photographie en détail, en ligne http://sirismm.si.edu/naa/baegn/gn_00094.jpg 
antinaturelle, la tête semble singulièrement petite ce qui, joint aux habits qui paraissent trop grands, donne une sensation de manque de tempérament, d'infériorité. Son regard est perdu, comme absent. En tout cas, ce portrait ne reflète pas I'homme intelligent, collaborateur et interprète d'anthropologues?'

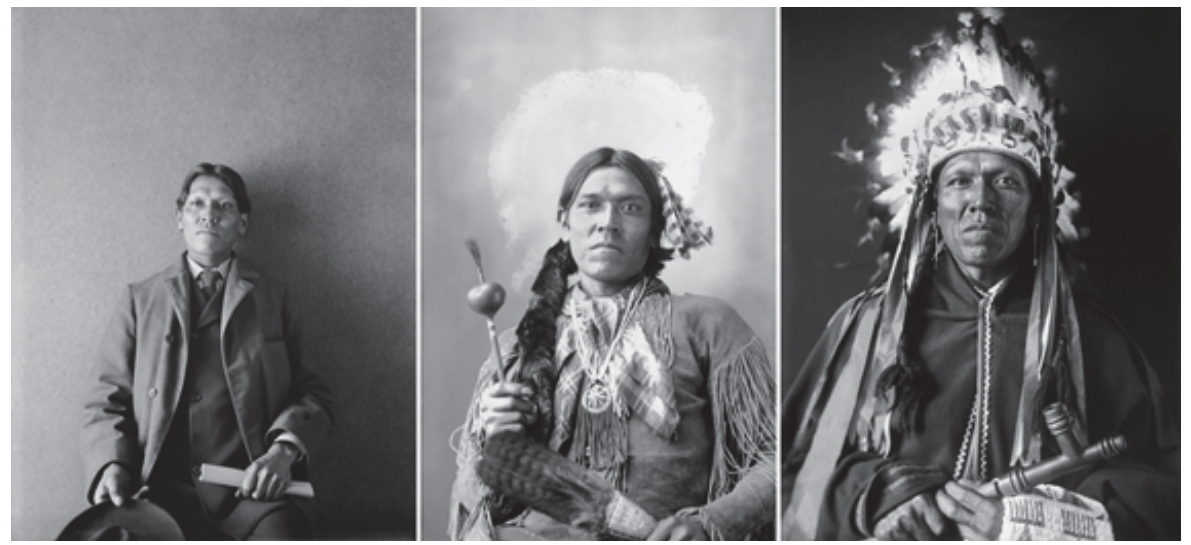

Portraits de Hitouunnen, aussi connu comme Cleaver Warden. Auteurs de gauche à droite: W. Dinwiddie; F.A. Rinehart; D. W. Gill.

Source: National Anthropological Archives, Smithsonian Institution

Dans le deuxième portrait, pris trois ans plus tard à Omaha, on peut découvrir que Warden n'était ni difforme, ni vulnérable : sa posture est naturelle et détendue, et il répond au regard du photographe. Il est donc aussi l'auteur de son propre portrait qui ne ressemble en rien à l'antérieur³.

Le troisième portrait, pris en 1909 par De Lancey Gill, a un but purement anthropologique, et quoiqu'il ne soit pas aussi répulsif que le premier, il prétend mettre en relief les traits caractéristiques de la race, effaçant d'une certaine manière l'homme. L'attitude corporelle est rigide, toutefois on observe que le regard que Warden renvoie au photographe est rempli d'ironie ${ }^{4}$. D'après ce que nous lu de sa biographie, on pourrait dire qu'il était anthropologue lui-même et donc qu'il était absolument conscient du but de ce portrait. Nous n'avons aucun doute que ce regard veut transmettre un message. Lequel ?

Selon Barthes, dans le portrait «quatre imaginaires s'y croisent [...]. Je suis celui que je me crois, celui que je voudrais qu'on me croie, celui que le photographe me croit, et celui dont il se sert pour exhiber son art » (1980:11). Ainsi, nous nous aventurons à soutenir l'opinion que dans le troisième portrait, si bien Warden, apparemment, se plie au désir du photographe de poser comme un «spécimen Arapaho », à travers du regard, il nie au photographe d'être celui qu'il croit, et est celui qu'il se croit, un homme intelligent capable d'ironie. Quant au premier portrait, on y trouve que deux imaginaires : celui du photographe qui croit que Warden est simplement un spécimen d'homme inférieur et sans qualité humaine et dont il se sert pour gonfler une collection anthropologique.

\subsection{Ohiyesa, Charles Eastman 1858-1939}

Ohiyesa, un Sioux Santee baptisé Charles Alexander Eastman, qui parlait sioux et anglais parfaitement et avait étudié grec, latin, français et allemand, fut le premier indien qui se diplôma comme docteur en médecine en 1890. II travailla comme interprète pour le Bureau of Indian Affairs et auprès du gouvernement, et contre sa volonté, il n'exerça presque pas sa profession de médecin (Karttunen, 1994). Aujourd'hui, Eastman est un écrivain reconnu dont les livres sont encore réédités avec succès.

\footnotetext{
2 En ligne http://sirismm.si.edu/naa/baegn/gn_00091.jpg

${ }^{3}$ En ligne http://sirismm.si.edu/naa/baegn/gn_00097.jpg

${ }^{4}$ En ligne http://sirismm.si.edu/naa/60/02908602.jpg
} 


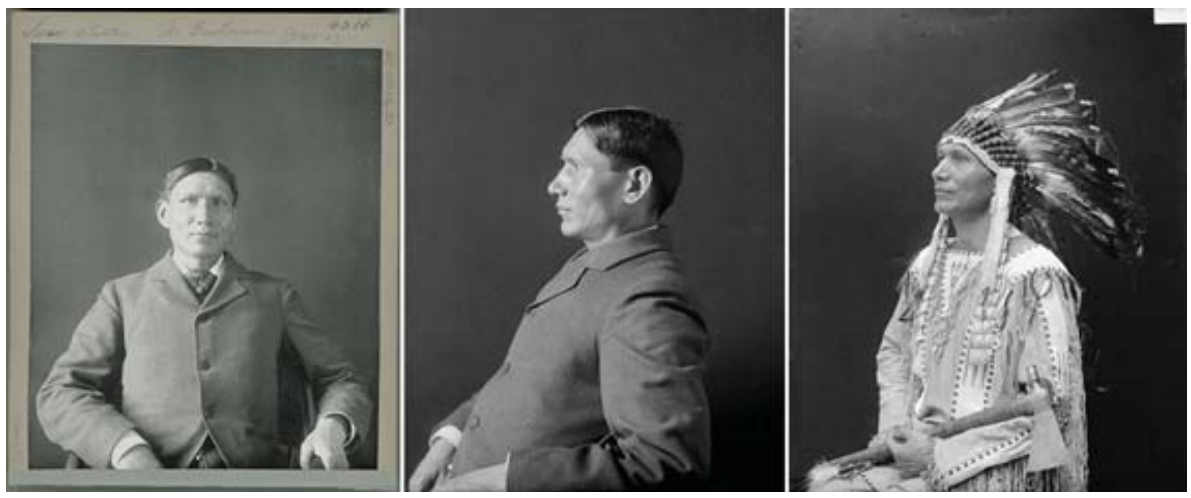

Portraits d'Ohiyesa, Dr Charles Alexander Eastman. Auteurs de gauche à droite: W. Sawyer ; W. Sawyer ; D.W. Gill.

Source: National Anthropological Archives, Smithsonian Institution

Les deux premiers portraits datent de 1897 et le troisième de 1913, donc Eastman était déjà docteur en médecine au moment de poser pour ces photos. Dans le premier portrait de Wells Sawyer nous avons l'impression, comme dans le cas de Werden, que la proportion entre la tête est le corps n'est pas correcte, et quoique Eastman regarde vers l'appareil, son regard est lointain, pensif ${ }^{5}$. Le deuxième portrait est un parfait exemple de l'application de la méthode photographique anthropométrique : la position du corps est forcée, car le but est de photographier la tête. Le troisième, par contre, semble plus naturel, si on le compare avec les deux premiers, mais c'est un portrait impersonnel, rigide, antipathique qui transmet une grande froideur. Le regard perdu d'Eastman dénote un besoin d'abstraction de cette situation vraisemblablement vexante ${ }^{6}$.

Dans aucun de ces trois portraits, nous pouvons reconnaître le docteur, l'écrivain, et l'étudiant brillant. En regardant ces photos, nous ne pouvons pas non plus apercevoir le sioux, car en croire les témoignages de personne qui ont vécu entre eux, comme Mary Eastman, l'épouse de Seth Eastman le grand-père de Charles, et Elaine Goodale, la femme d'Eastman, les Sioux sont des hommes séduisants de haute taille (Karttunen, 1994:152-53). Pour le faire, il nous faut contempler d'autres portraits, comme par exemple celui du Knox College datant de 188017, ou celui du Dartmouth College ${ }^{8}$.

\subsection{David Zephier}

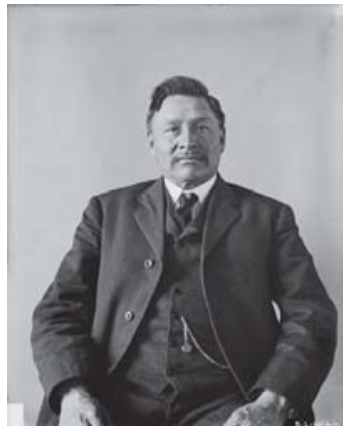

\footnotetext{
${ }^{5}$ En ligne http://sirismm.si.edu/naa/baegn/gn_03463.jpg

${ }^{6}$ The Newberry Library, en ligne http://publications.newberry.org/frontiertoheartland/items/show/164

${ }^{7}$ World Wisdom, en ligne http://www.worldwisdom.com/public/slideshows/view.aspx?SlideShowID=3\&SlideDetaillD=4

${ }^{8}$ Portraits de Fukuzawa, en ligne http://photographs.prm.ox.ac.uk/jpeg_1200s/1951_11_63_80-0.jpg

http://photographs.prm.ox.ac.uk/jpeg_1200s/1951_11_63_81-0.jpg

http://photographs.prm.ox.ac.uk/jpeg_1200s/1951_11_63_82-0.jpg
} 
David Zephier était un métis Sioux Dakota, né en 1857. Interprète professionnel, il parlait couramment français, dakota et anglais. II travailla pour les délégations Sioux Yankton qui visitaient Washington (Maroukis et Bruguier, 2005: 66), et il fut de témoin direct de négociations historiques.

Dans le portrait, ci-contre, de D.W. Gill (1907), on peut observer de nouveau ce manque de proportion entre la tête et corps, surtout dans ce cas, avec les mains. Malgré tout, il nous semble que David Zephier réussit à ne pas disparaître derrière le portrait anthropométrique montrant une grande dignité et aplomb, en regardant de face le photographe.

Dans la page suivante, nous avons un portrait de Gill, de cinq chefs Sioux et de leur interprète, David Zephier. La photographie n'est vraiment pas artistique, peu soignée même, remarquons la toile qui sert de fond. Mais ce qui nous intéresse le plus de relever est que Zephier est le seul habillé avec un complet. Les chefs, eux, portent leurs costumes traditionnels, et nous savons grâce aux photographies de l'époque que ceci était exceptionnel. Donc, nous pouvons en déduire qu'ils le firent sur demande de Gill. Aussi, nous parait-il évident que ce fait n'est pas casuel, et nous supposons que Zephier choisit sa tenue consciemment. Pourquoi ? Nous essayerons d'y répondre en peu plus en avant.

\subsection{Yukichi Fukuzawa}

Fukuzawa est le plus célèbre de tous nos interprètes. Né à Osaka en 1835, travailla comme interprète de néerlandais et d'anglais pour le Bureau des affaires étrangères. En 1862, il voyagea en Europe comme interprète de la première mission diplomatique japonaise, et c'est à Paris que Potteau lui fit trois portraits ${ }^{9}$. De retour dans son pays, il fonda l'université de Keio. Traducteur et écrivain prolifique, il publia des livres et des articles sur politique intérieure et internationale, économie politique, éducation et politique éducative, problèmes de morale publique et, notamment, droits des femmes (Nishikawa, 1993). Le portrait plus célèbre de Fukuzawa et celui qui figure sur les billets de 10000 yens, car il est considéré un des fondateurs du Japon moderne.

II faut reconnaître que les photographies de Potteau, comparée à celles prises par ses homologues américains sont de meilleures qualités et au moins ne déforme pas la figure. Mais de toute façon, elles ont quelque chose de déconcertant, surtout celle de profil. Peut-être est-ce le contraste entre la dignité de l'attitude Fukuzawa et le regard investigateur du photographe. Il est aussi probable que Potteau fût intimidé par les armes que portaient Fukusawa.

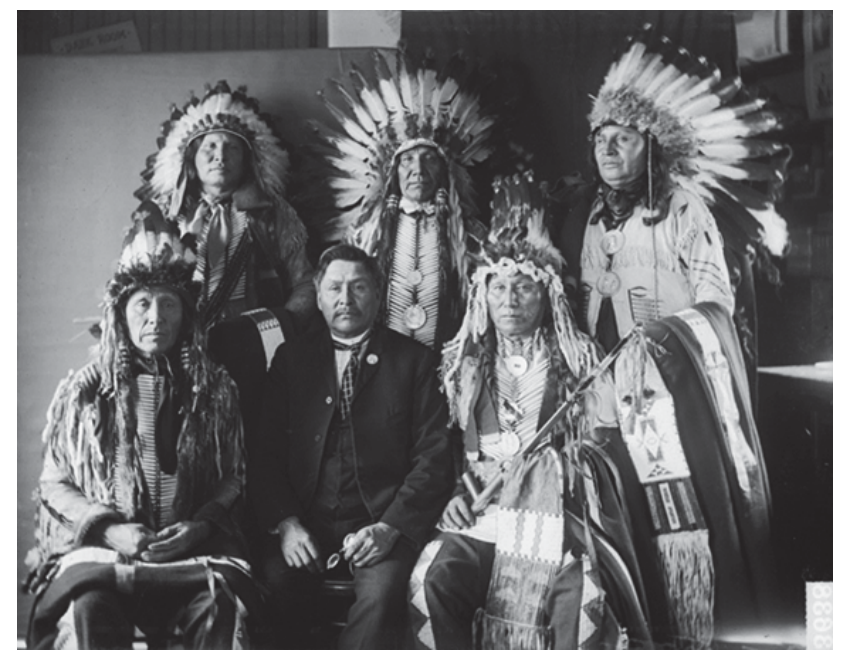

De gauche à droite, debout : Eagle Track, Charging Bear, Hollow Horn; assis : Shooting Hawk, David Zephier, Yellow Thunder.

Source: D.W. Gill. National Anthropological Archives, Smithsonian Institution 


\section{Images d'interprètes : perception de l'Autre}

Les résultats de l'analyse visuelle des photographies sont accablants. II est évident que les anthropologues ne perçoivent pas les interprètes, ils ne voient que le spécimen d'une race différente à la sienne. Ces portraits anthropologiques annulent d'une certaine façon la personne, il ne reste que les traits physiques soi-disant d'une race, d'une ethnie ou d'une tribu. Comment était-il possible que ces personnages dont les qualités intellectuelles étaient indéniables, deviennent invisibles, et qu'ils soient rabaissés à des types humains ? Comment était-ce possible que Warden, étroit collaborateur d'anthropologue, collègue même, soit dégradé, lui aussi, à un spécimen ? II va sans dire que nous pensons de même pour n'importe quelle femme ou homme.

Les anthropologues légitimaient cette pratique expérimentale par la différence : «Si je peux les observer c'est parce qu'ils ne sont pas comme moi » (Affergan, 1987:228). Â ceci s'ajoute leur vision ethnocentrique qui postule: " dans notre endogroupe, nous sommes tous les mêmes ; les Autres (les membres de l'exogroupe) sont tous pareils ; mais les Autres sont très différents de Nous » (Staszak, 2008: 11). Mais qui formait cet endogroupe ? Buffon avait établi au XVIIIle siècle qu'il était constitué par l'homme vivant entre le $40^{\mathrm{e}}$ et $50^{\mathrm{e}}$ degré, et toujours selon Buffon, la zone où se trouvaient les hommes les plus beaux et les mieux faits (Affergan, 1987:232).

II est clair ici que les anthropologues se considèrent membres de cet endogroupe aux qualités physiques supérieures, et donc à leurs yeux il n'y pas de beauté chez les Autres, ce qui expliquerait parfaitement le mépris esthétique de la plupart des portraits étudiés. Mais ce qui est pire, c'est que ces photographies qui reflètent leurs préjugés esthétiques, ne sont plus une empreinte de la réalité, ni un document scientifique, comme ils prétendaient. On ne peut même pas les considérer des portraits, car comme nous l'avons démontré, ils défigurent le modèle.

Ils étaient donc incapables de voir les traits physiques de l'Autre, mais ceci n'explique pas pourquoi, pouvant se communiquer dans leur langue avec les interprètes, ils ne percevaient pas leurs qualités intellectuelles. Selon Affergan, toute différence, en opposant, nie quelque chose de quelque chose, elle désidentifie (1987 :226). Il est intéressant ici de remarquer que les anthropologues et les ethnologues désignent les indigènes qui leur sert d'interprètes sous le terme d'informateur. Cette distinction entre interprète et informateur est révélatrice sur leur façon de s'approcher à l'Autre : à travers de l'interprète, on le comprend; à travers l'informateur, on l'observe. L'informateur, agent passif, se limite à transmettre une information, l'interprète, par contre, adapte et rend compréhensible un discours.

Est-ce que l'anthropologie actuelle a changé ? Nous n'avons pas assez de connaissances sur cette matière ni pour l'affirmer, ni le nier. Ce que nous avons observé est, que malheureusement, les collections digitales des archives anthropologiques perpétuent cette perception. Si on regarde un portrait d'une personne dont on ne connaît que le nom, la tribu ou la nationalité, et peut-être la profession, il est déjà difficile de percevoir l'Autre au-delà des stéréotypes. Mais si à cela, on ajoute une photographie anthropométrique, l'Autre s'efface et le sujet devient une caricature, ou s'il a la chance de porter des habits tribaux, il se transforme en une créature exotique. À notre avis, dans les deux cas, c'est infâme.

Pour l'éviter, il faut re-contextualiser la photographie en : explicitant son objectif anthropologique et son caractère anthropométrique ; l'accompagnant d'une brève biographie du sujet; et mentionnant, s'ils existent, d'autres portraits. Alors l'Autre peut surgir et la perception de la photographie change complètement comme nous l'avons montré dans ce travail. Même Riwa, le modèle de Portman, dont nous ne connaissons aucun détail, se transforme de sauvage nu en membre essentiel d'une communauté, si on s'attarde à s'imaginer ce qu'implique son labeur d'interprète.

Quoique ceci, parfois, n'est pas suffisant, et ces photographies ne réussissent pas à échapper à leur triste destin. Comme c'est le cas des photographies de Potteau. Le Musée Pitt Rivers organisa une exhibition en 2011 qui contextualisait les photographies avec, entre autres, une brève biographie de chaque personnage photographié, cependant il l'intitula The last Samurai : Jacques-Philippe Potteau's Photographs of the Japanese Missions to Europe, 1862 and 1864. Mis à part que c'est une grande inexactitude parler des derniers Samurais, on retrouve une fois de plus, le regard qui réduit l'Autre à une anecdote exotique.

Mais est-ce que les interprètes qui apparaissent dans ces photographies étaient-ils conscients de tout cela ? Nous en sommes convaincus. Ces interprètes travaillaient en contact direct avec les interlocuteurs, et ils pouvaient donc déceler le regard de l'autre. Nous en avons un indice dans ce travail dans le portrait du groupe de chefs indiens et de leur interprète. Nous sommes sûrs que Zephier ne porte pas le costume tribal pour essayer d'éliminer toutes les différences possibles, et de donner une image familière à l'homme occidental, de personne cultivée et de professionnel et ainsi faire passer à un deuxième plan son origine indienne.

Nous pouvons conclure que les interprètes étaient perçus par les anthropologues du XIXe comme des sauvages ; sauvages apprivoisés dans le cas des interprètes Sioux; sauvage exotique dans celui de l'interprète japonais ; tout 
simplement sauvage quant à Riwa l'interprète des îles Andaman. Aujourd'hui, notre devoir est d'éviter de perpétuer cette image dans les collections, les expositions et les musées modernes.

\section{Références}

A Manual of Ethnological Inquiry. (1854).Journal of the Ethnological Society of London (1848-1856), 3, 193-208. doi:10.2307/3014143

Affergan, F. (1987). Exotisme et altérité: essai sur les fondements d'une critique de l'anthropologie (Vol. 48). Paris: Presses Universitaires de France-PUF.

Anderson, J. D. (1997). Introduction. In G. G. A. Dorsey \& A. A. L. Kroeber (Eds.), Traditions of the Arapaho. Lincoln: University of Nebraska Press.

Barthes, R. (1980). La chambre claire : note sur la photographie. Paris: Cahiers du cinéma.

Bourdieu, P., Boltanski, L., Castel, R., \& De Vendeuvre, P. (1965). Un art moyen. Paris: Minuit.

Brisset Martin, D. E. (1999). Acerca de la fotografía etnográfica. Ga, 15(Artículo 11).

Broca, P. (1865). Instructions générales pour les recherches et observations anthropologiques (anatomie et physiologie). (p. 1 vol.). Paris: V. Masson et fils. En ligne http://catalogue.bnf.fr/ark:/12148/cb301636125

Jehel, P.-J. (1995). Photographie et anthropologie en France au XIXe siècle. Université Paris VIII. Saint-Denis. En ligne http:/lwww.a-me-r.com/Recherches/dea/photo_anth19e.pdf

Jehel, P-J. (2000). Une illusion photographique. Journal des anthropologues.Association française des anthropologues, 81, 47-70.

Karttunen, F. E. (1994). Between Worlds: Interpreters, Guides and Survivors. New Brunswick: Rutgers University Press.

Lachat Leal, C. (2013). Retratos de intérpretes en archivos fotográficos digitales ( 1839 - 1914 ). Actas del VI Congreso AIETI. Las Palmas de Gran Canaria, sous presse. En ligne http://digibug.ugr.es/handle/10481/24236

Leonard, J. W., \& Marquis, A. N. (1898). Who's who in America : a biographical dictionary of notable living men and women of the United States. Chicago, Illinois: A.N. Marquis Co. [etc.]. En ligne http://www.archive.org/details/whoswhoinameric00incgoog

Maresca, S. (1996). La photographie, un miroir des sciences sociales. Paris: Editions L'Harmattan.

Maroukis, T. C., \& Bruguier, L. R. (2005). Peyote And the Yankton Sioux: The Life And Times of Sam Necklace. Norman: University of Oklahoma Press.

Nishikawa, S. (1993). Fukuzawa yukichi (1835-1901). Perspectives: revue trimestrielle d'éducation comparée, XXIII(3-4), 501-515. En ligne http://www.ibe.unesco.org/publications/ThinkersPdf/fukuzawf.pdf

Portman, M. V. (1896). Photography for Anthropologists. Journal of the Anthropological Institute of Great Britain and Ireland, 75-87. En ligne http://www.jstor.org/stable/2842469

Scherer, J. C. (2006). A Danish Photographer of Idaho Indians: Benedicte Wrensted. Norman: University of Oklahoma Press.

Sheptytsky-Zäll, J. (2009). Collection anthropologique du Muséum de Paris: ethnographic portraits by Jacques-Philippe Potteau. Ryerson University. En ligne http://digitalcommons.ryerson.ca/dissertations/515

Smithsonian American Art Museum. (n.d.). Frank A. Rinehart. En Ligne http://americanart.si.edu/collections/search/artist/?id=6740, consulté le 11 mars 2013.

Staszak, J. (2008). Qu' est-ce que l'éxotisme ? Le Globe, 148, 7-30.

Trutat, E. (1892). La photographie appliquée à l'histoire naturelle / par M. Trutat. Paris: Gauthier-Villars et fils. En ligne http://www.cineressources.net/images/ouv_num/061.pdf

\section{Photographies}

Dinwiddie, W. (1895). Portrait (Front) of He-Tu-Na or Hitunena, Called Cleaver Warden, Interpreter and Informant MAR 1895. BAE GN 00094 06089400, National Anthropological Archives, Smithsonian Institution. En ligne http://www.siris.si.edu

Gill, L. W. (1905). Delegation to Washington, March 1905 Mar 1905. Negative 3638, National Anthropological Archives, Smithsonian Institution. En ligne http://www.siris.si.edu

Gill, L. W. (1907). Two Kettle Dakota man, David Zephier 1907. Negative 3324 A, National Anthropological Archives, Smithsonian Institution. En ligne http://www.siris.si.edu

Gill, L. W. (1909). Portrait (Front) of He-Tu-Na or Hituena, Called Cleaver Warden, Interpreter and Informant, in Native Dress with Headdress and Holding Pipe and Bag JUN 1909. BAE GN 00097 06089700, National Anthropological Archives, Smithsonian Institution. En ligne http://www.siris.si.edu

Gill, L. W. (1913). Portrait of Ohiyesa or The Winner. Known as Dr. Charles Alex. Eastman, physician and author. BAE GN 3463, National Anthropological Archives, Smithsonian Institution. En ligne http://www.siris.si.edu

Potteau, J.-P. (1862a). Fukuzawa Yukichi. 1951.11.63.80, Pitt Rivers Museum. En ligne http://www.prm.ox.ac.uk/

Potteau, J.-P. (1862b). Fukuzawa Yukichi. 1951.11.63.81,Pitt Rivers Museum. En ligne http://www.prm.ox.ac.uk/

Potteau, J.-P. (1862c). Fukuzawa Yukichi. 1951.11.63.82, Pitt Rivers Museum. En ligne http://www.prm.ox.ac.uk/

Rinehart, F. A. (1898). Portrait (Front) of Hitunena, Called Gros Ventre Man or Cleaver Warden, in Peyote Costume with Peyote Feather Fan and Peyote Rattle 1898. Photo Lot 60: Bae Ref Album 11: Arapaho/Cheyenne 0, National Anthropological Archives, Smithsonian Institution. En ligne http://www.siris.si.edu

Sawyer, W. (1897a). Portrait (Front) of Ohiyesa (The Winner), Called Dr Charles Alexander Eastman, Physician, Author, and Brother of 
Rev John Eastman MAR 1897. Photo Lot 60: Bae Ref Album 9: Osage/Shoshone/Nez, National Anthropological Archives, Smithsonian Institution. En ligne http://www.siris.si.edu

Sawyer, W. (1897b). Portrait (Profile) of Ohiyesa (The Winner), Called Dr Charles Alexander Eastman, Physician, Author, and Brother of Rev John Eastman MAR 1897. Photo Lot 60: Bae Ref Album 9: Osage/Shoshone/Nez, National Anthropological Archives, Smithsonian Institution. En ligne http://www.siris.si.edu 